\title{
MINKOWSKI-ADDITIVE MULTIMEASURES, MONOTONICITY AND SELF-SIMILARITY
}

\author{
DAVIDE LA TORRE ${ }^{1}$ AND FRANKLIN MENDIVIL ${ }^{2}$ \\ ${ }^{1}$ Department of Economics, Business and Statistics, University of Milan, Italy; ${ }^{2}$ Department of Mathematics and \\ Statistics, Acadia University, Wolfville, Nova Scotia, Canada. \\ e-mail: davide.latorre@unimi.it, franklin.mendivil@acadiau.ca \\ (Accepted August 31, 2011)
}

\begin{abstract}
We discuss the main properties of positive multimeasures and we show how to define a notion of self-similarity based on a generalized Markov operator.
\end{abstract}

Keywords: cone-positive multimeasure, fractal transforms, positive multimeasures, self-similarity.

\section{INTRODUCTION}

In the first part of this paper we introduce two different notions of positive multimeasures, namely positive multimeasures and cone-positive multimeasures, and we analyze some mononicity properties of these classes of multimeasures. In the second part we introduce a definition of self-similar multimeasure based on a Markov operator.

There are many applications of set functions to different areas such as mathematical economics, decision theory and social sciences; for this reason, many variations on and extensions of measures have been provided in the literature including, for instance, subadditive and superadditive set functions, submeasures, null-additive set functions and so on. The notion of multimeasure (Vind, 1964; Debreu and Schmeidler, 1967; Artstein, 1972) is one of these possible generalizations which consider set-valued set functions instead of set functions. Some practical motivations behind this kind of mathematical structures can be found, for instance, in mathematical economics, when coalitions are considered as primitive economic units (Vind, 1964; Debreu and Schmeidler, 1967) or in the theory of capacities (Choquet, 1953). There has been a wealth of work on multimeasures and generalized measures, both theoretical and in applications (Choquet, 1953; Vind, 1964; Debreu and Schmeidler, 1967; Brooks, 1968; Artstein, 1972; Hildenbrand, 1974; Drewnokski, 1976; Pap, 1991; Kan, 1992; Alo et al., 1980; Guo and Zhang, 2004).

Our purpose in this paper is to provide a useful class of multimeasures for modelling of images and information derivable from images. Images are often modeled as functions or measures and implicitly these are most often positive. In the case of measures, restricting to positive measures greatly simplifies the technical details as spaces of positive measures have nicer properties than spaces of signed measures (one example is the relationship between bounded variation of a positive measure $\mu$ and the boundedness of the total mass, $\mu(\Omega)$, of $\mu$ ). Thus we examine two different classes of positive multimeasures. Many images exhibit approximate self-similarity and this structure has proven very useful in applications in image compression, representation and analysis (Hutchinson, 1981; Barnsley and Demko, 1985; Barnsley et al., 1985; Barnsley, 1989; Forte et al., 1999; Forte and Vrscay, 1999; Iacus and La Torre, 2005a;b; Kunze et al., 2007; 2008; 2012; La Torre and Mendivil, 2008; 2009; La Torre et al., 2009). For instance, in fractal image coding based on Iterated Function Systems (IFS) and their generalization, the self-similar attractor is defined in terms of a compact set and a positive measure supported on it. The positive measure is the unique fixed point of a Markov operator defined on a suitable space of positive measures. The main idea behind this paper is to extend this approach to the case of multimeasures, by defining an appropriate complete space of multimeasures and a contractive Markov operator which will be used to introduce a notion of self-similarity in this class.

The paper is organized as follows: next section reviews the definition of (Minkowski additive) multimeasures and introduces a metric space of multimeasures which is complete once an extension of the Monge-Kantorovich metric is given. The third section introduces a weak notion of positive multimeasure; for a multimeasure $\Phi$ to be positive it is enough that 0 belongs to $\Phi(A)$ for all measurable sets $A$. In the fourth section a stronger notion of positive multimeasure is provided; in this context, 
a multimeasure $\Phi$ is positive if $\Phi(A) \subseteq P$, for all measurable sets $A$, where $P$ is an ordering cone. Finally a definition of self-similarity based on a notion of generalized Markov operator and some examples are provided in the last section.

\section{ADDITIVE MULTIMEASURES}

Consider a nonempty set $\Omega$ and a $\sigma$-algebra $\mathscr{F}$ on $\Omega$. A set-valued measure or multimeasure on $(\Omega, \mathscr{F})$ is a function (for more on multimeasures see Artstein, 1972)

$$
\Phi: \mathscr{F} \rightarrow\left\{K \subset \mathbb{R}^{m}: K \neq \emptyset\right\},
$$

which for any sequence of disjoint sets $A_{i} \in \mathscr{B}$ satisfies

$$
\Phi\left(\bigcup_{i=1}^{\infty} A_{i}\right)=\sum_{i=1}^{\infty} \Phi\left(A_{i}\right) .
$$

The right side is the infinite Minkowski sum given by

$$
\sum_{i} K_{i}=\left\{\sum_{i} k_{i}: k_{i} \in K_{i}, \sum_{i}\left|k_{i}\right|<+\infty\right\} .
$$

For $A \subset \mathbb{R}^{m}$ and $q \in \mathbb{R}^{m}$ recall that

$$
\operatorname{supp}(q, A)=\sup \{q \cdot x: x \in A\}
$$

defines the support function. For a multimeasure $\Phi$ let

$$
\Phi^{q}(B)=\sup \{q \cdot x: x \in \Phi(B)\}=\operatorname{supp}(q, \Phi(B)) .
$$

Then $\Phi^{q}(\cdot)$ is a signed measure with values in $(-\infty, \infty]$. Versions of the Radon-Nikodym theorem have been proved for multimeasures (Artstein, 1972). Let $\mathbb{H}_{c}\left(\mathbb{R}^{m}\right)$ be the space of all compact and convex subsets of $\mathbb{R}^{m}$. In the following we work with multimeasures defined on the Borel $\sigma$-algebra $\mathscr{F}$ of a complete metric space $\Omega$ taking values in $\mathbb{H}_{c}\left(\mathbb{R}^{m}\right)$. Let $Q, K \in \mathbb{H}_{c}\left(\mathbb{R}^{m}\right)$ with $Q \subseteq K$. We define $\mathscr{M}_{Q, K}\left(\Omega, \mathbb{R}^{m}\right)$ to be the set of all Minkowski $\sigma$ additive multimeasures $\Phi$ on $\Omega$ which take values in $\mathbb{H}_{c}\left(\mathbb{R}^{m}\right)$ and such that

1. $\Phi(\Omega)=Q$

2. There is some set $D \in \mathbb{H}_{c}\left(\mathbb{R}^{m}\right)$ and $a \in \Omega$ so that $\int_{\Omega} f(x) \mathrm{d} \Phi(x) \subseteq D$ for all $f \in \operatorname{Lip}_{1}(\Omega)$ with $f(a)=$ 0 .

3. $\Phi(A) \subseteq K$ for all $A$.

Let $S_{1}=\left\{x \in \mathbb{R}^{m}:\|x\|=1\right\}$ be the unit sphere in $\mathbb{R}^{m}$. We define the following metric on $\mathscr{M}_{Q, K}\left(\Omega, \mathbb{R}^{m}\right)$,

$$
\hat{d}_{M}\left(\Phi_{1}, \Phi_{2}\right)=\sup _{p \in S_{1}} d_{M}\left(\Phi_{1}^{p}, \Phi_{2}^{p}\right) .
$$

In this, $d_{M}(\mu, v)$ is the natural extension of the usual Monge-Kantorovich metric between two probability measures (see Kunze et al., 2012). It can be proved that the space $\left(\mathscr{M}_{Q, K}\left(\Omega, \mathbb{R}^{m}\right), \hat{d}_{M}\right)$ is a complete metric space (Kunze et al., 2012).

\section{POSITIVE MULTIMEASURES}

We say that a multifunction $F: D \subseteq \mathbb{R}^{n} \rightrightarrows$ $\mathbb{R}^{m}$ is positive if $0 \in F(x)$ for all $x \in D$. In a similar way one can define positive multimeasure; given a measurable space $(\Omega, \mathscr{F})$ a multimeasure $\Phi: \mathscr{F} \rightrightarrows X$ is said to be positive if $0 \in \Phi(A)$ for all $A \in \mathscr{F}$. Let us define $\mathscr{M}_{Q, K}^{+}\left(\Omega, \mathbb{R}^{m}\right)$ be the subspace of positive multimeasures; using the completeness of $\mathscr{M}_{Q, K}\left(\Omega, \mathbb{R}^{m}\right)$ it is easy to show that $\left(\mathscr{M}_{Q, K}^{+}\left(\Omega, \mathbb{R}^{m}\right), \hat{d}_{M}\right)$ is a complete metric space.

The following result is easy to prove.

Proposition 1. If $\Phi$ is a positive multimeasure then $\Phi^{p}$ is a positive measure for all $p \in S^{1}$.

Given two positive multimeasures $\Phi: \mathscr{F} \rightrightarrows X$ and $\psi: \mathscr{F} \rightrightarrows X$ we say that $\Phi$ is absolutely continuous with respect to $\psi$ if $\Phi^{p}$ is absolutely continuous with respect to $\psi^{p}$ for all $p \in S^{1}$ and in this case we write $\Phi \ll \psi$. If $\Phi$ is absolutely continuous with respect to $\psi$ and $\psi(A)=\{0\}$ then $\Phi(A)=\{0\}$. The following result provides a list of some simple properties of positive multi-functions and positive multimeasures. Let $F$ be a positive multi-function and $\Phi$ be a positive multimeasure. Then:

1. For all $f: \Omega \rightarrow \mathbb{R}$ we have $f(x) F(x)$ is a positive multi-function.

2. For all $p \in \mathbb{R}^{m}$, the real-valued function $F^{p}$ defined by $F^{p}(x)=\operatorname{supp}(p, F(x))$ is nonnegative.

3. $A \subseteq B$ implies that $\Phi(A) \subseteq \Phi(B)$. Thus, $\Phi(A) \subseteq$ $\Phi(\Omega)$ for all $A$.

4. For all $p \in \mathbb{R}^{m}$, the signed measure $\Phi^{p}$ is a positive measure.

5. If $\mu$ is a positive measure then the multimeasure $\Phi$ defined by $\Phi(A)=\int_{A} F(x) \mathrm{d} \mu(x)$ is a positive multimeasure.

6. If $0 \leq f(x) \leq g(x)$, then $\int_{\Omega} f(x) \mathrm{d} \Phi(x) \subseteq$ $\int_{\Omega} g(x) \mathrm{d} \Phi(x)$.

Proposition 2. Suppose that $F$ is a multifunction, $F(x)$ is a convex set for all $x, \mu$ is a positive measure and $\Phi$ defined by

$$
\Phi(A)=\int_{A} F(x) \mathrm{d} \mu(x)
$$

is a positive multimeasure. Then $F$ is $\mu$-a.e. a positive multifunction. 
Proof. Suppose that the conclusion is false. Then there is some set $A \subseteq \Omega$ with $\mu(A)>0$ and $0 \notin F(x)$ for all $x \in A$. Let $p_{n}$ be a countable dense sequence in $S^{1}$. For each $x \in A$, there is some $n \in N$ so that $\operatorname{supp}\left(p_{n}, F(x)\right)<0$. Thus, with $A_{n}^{m}=\{x \in A$ : $\left.\operatorname{supp}\left(p_{n}, F(x)\right)<-1 / m\right\}$ we have

$$
A=\bigcup_{n, m \in \mathbb{N}} A_{n}^{m}
$$

and so there are $n, m$ with $\mu\left(A_{n}^{m}\right)>0$. However, then this means that

$$
\begin{aligned}
& 0>-\frac{\mu\left(A_{n}^{m}\right)}{m} \geq \int_{A_{n}^{m}} \operatorname{supp}\left(p_{n}, F(x)\right) \mathrm{d} \mu(x)= \\
& \operatorname{supp}\left(p_{n}, \int_{A_{n}^{m}} F(x) \mathrm{d} \mu(x)\right)=\operatorname{supp}\left(p_{n}, \Phi\left(A_{n}^{m}\right)\right),
\end{aligned}
$$

and thus $0 \notin \Phi\left(A_{n}^{m}\right)$ which contradicts the fact that $\Phi$ is positive. Thus $F$ must be a positive multifunction.

Corollary 1. If $\Phi$ is a positive multimeasure which is absolutely continuous with respect to the positive measure $\mu$, then the Radon-Nikodym derivative $F(x)$ of $\Phi$ with respect to $\mu$ is a positive multifunction.

We mention that in Proposition 2 it also works if $\mu$ is a signed measure and not just a positive measure. To see this, one just decomposes $\Omega$ into positive and negative sets and apply the same proof to each part.

Proposition 3. Suppose that $\Phi$ is a positive multimeasure and $f$ is a real-valued function on $\Omega$. Then $v$ defined by

$$
v(A)=\int_{A} f(x) \mathrm{d} \Phi(x)
$$

is a positive multimeasure.

Proof. First suppose that $f(x) \geq 0$ for all $x$. Then for any $A$ we have

$$
\begin{aligned}
0 \leq & \int_{A} f(x) \mathrm{d} \Phi^{p}(x)= \\
& \quad \operatorname{supp}\left(p, \int_{A} f(x) \mathrm{d} \Phi(x)\right)=\operatorname{supp}(p, v(A)),
\end{aligned}
$$

for any $p \in S^{1}$. Thus $0 \in v(A)$ and so $v$ is positive in this case. If $f(x)<0$ for all $x$, we just use the fact that $\operatorname{supp}(p, f(x) \Phi(A))=\operatorname{supp}(-p,|f(x)| \Phi(A))$ and get the same result. Now, if $f$ is a general real-valued function, let $P=\{x \in \Omega: f(x) \geq 0\}$ and $N=\{x$ : $f(x)<0\}$. Then $A=(A \cap N) \cup(A \cap P)$ and so $v(A)=$ $v(A \cap N)+v(A \cap P)$. However, by the argument from the first part (where $f$ is positive) we see that $0 \in$ $v(A \cap N)$ and $0 \in v(A \cap P)$ and so $0 \in v(A)$ and thus $v$ is a positive multimeasure.
We now deal with type of monotone convergence for positive multimeasures.

Proposition 4. Let $\Phi_{n}$ be a sequence of positive multimeasures with $\Phi_{n}(A) \subseteq \Phi_{n+1}(A)$ for all $n$ and $A \in \mathscr{F}$. Then $\Phi$ defined by

$$
\Phi(A)=\overline{\bigcup_{n} \Phi_{n}(A)}
$$

is also a positive multimeasure.

Proof. The only non-trivial property is countable additivity. Thus, suppose $\left\{A_{m}\right\}$ are disjoint sets in $\mathscr{F}$. Notice that $\sum_{m} \Phi_{n}\left(A_{m}\right) \subseteq \sum_{m} \Phi_{n+1}\left(A_{m}\right)$. Using this and the definition of countably additive, we see that $\zeta \in \sum_{m} \Phi\left(A_{m}\right)$ iff for all $\varepsilon>0$, there exists some $N \in \mathbb{N}$ and points $y_{i} \in \Phi\left(A_{m_{i}}\right)$ with $m_{i}<N$ so that $\left|\sum_{i} y_{i}-\zeta\right|<\varepsilon$. Further, this happens iff for all $\varepsilon>0$ there is some $N \in \mathbb{N}$ and $y_{i} \in \Phi_{n_{i}}\left(A_{m_{i}}\right)$ where $n_{i}<$ $N$ and $m_{i}<N$ so that $\left|\sum_{i} y_{i}-\zeta\right|<\varepsilon$. On the other hand, $\zeta \in \Phi\left(\cup_{m} A_{M}\right)$ iff for all $\varepsilon>0$ there is some $z \in \cup_{n} \Phi_{n}\left(\cup_{m} A_{m}\right)$ with $|z-\zeta|<\varepsilon$ which happens iff for all $\varepsilon>0$ there is some $N \in \mathbb{N}$ and a points $y_{i} \in \Phi_{n_{i}}\left(A_{m_{i}}\right)$ with $n_{i}<N$ and $m_{i}<N$ so that $\left|\sum_{i} y_{i}-\zeta\right|<\varepsilon$.

Proposition 5. Let $K \subseteq \mathbb{R}^{m}$ be compact and $\Phi_{n}$ be a sequence of multimeasures with $\Phi_{n}(A) \subseteq \Phi_{n+1}(A) \subseteq$ $K$ for all $n$ and $A$. Then $\Phi$ defined by

$$
\Phi(A)=\overline{\bigcup_{n} \Phi_{n}(A)}=\lim _{n} \Phi_{n}(A)
$$

is a positive multimeasure, where the limit is taken in the Hausdorff distance. If $f$ is any $|\Phi|$-integrable nonnegative function on $\Omega$ then

$$
\int_{\Omega} f(x) \mathrm{d} \Phi_{n}(x) \nearrow \int_{\Omega} f(x) \mathrm{d} \Phi(x),
$$

where again the convergence is in the Hausdorff metric.

Proof. For any $A \in \mathscr{F}$ and $p \in \mathbb{R}^{m}$, we see that

$$
\begin{array}{r}
\Phi^{p}(A)=\operatorname{supp}(p, \Phi(A))=\operatorname{supp}\left(p, \overline{\bigcup_{n} \Phi_{n}(A)}\right) \\
=\sup _{n} \operatorname{supp}\left(p, \Phi_{n}(A)\right)=\operatorname{limsupp}_{n}\left(p, \Phi_{n}(A)\right) \\
=\lim _{n} \Phi_{n}^{p}(A) \leq \operatorname{supp}(p, K) .
\end{array}
$$

This means that for all $p$, we have that $\Phi^{p}$ is a well-defined measure and thus $\Phi$ is well-defined as a multimeasure with compact and convex values. Furthermore, $0 \in \Phi_{n}(A) \subseteq \Phi(A) \subseteq K$ for all $n$ and 
$A \in \mathscr{F}$. Thus $\Phi$ is a bounded positive multimeasure. Notice that we know

$$
\lim _{n} \Phi_{n}^{p}(A)=\Phi^{p}(A)
$$

for all $p \in \mathbb{R}^{m}$ and $A \in \mathscr{F}$ and that this trivially implies that for all simple functions $g$ we have

$$
\lim _{n} \int_{\Omega} g(x) \mathrm{d} \Phi_{n}^{p}(x)=\int_{\Omega} g(x) \mathrm{d} \Phi^{p}(x)
$$

for each $p$. We know $\left|\Phi_{n}(A)\right| \leq|\Phi(A)|$ for all $n$ and thus $f$ is also $\left|\Phi_{n}\right|$-integrable for any $n$. Since any selector for $\Phi_{n}$ is also a selector for $\Phi_{n+1}$ and for $\Phi$, for all $n$ we have

$$
\int_{\Omega} f(x) \mathrm{d} \Phi_{n}(x) \subseteq \int_{\Omega} f(x) \mathrm{d} \Phi_{n+1}(x) \subseteq \int_{\Omega} f(x) \mathrm{d} \Phi(x) .
$$

Further, $\int_{\Omega} f(x) \mathrm{d} \Phi(x) \subseteq \mathbb{R}^{m}$ is compact and convex. To show the reverse inclusion, suppose that it isn't true. That is, suppose that there is some

$z \in \int_{\Omega} f(x) \mathrm{d} \Phi(x) \quad$ but $\quad z \notin L:=\overline{\bigcup_{n} \int_{\Omega} f(x) \mathrm{d} \Phi_{n}(x)}$.

Then since $L$ is compact and convex, there is some $\varepsilon>0$ and a $p \in S^{1}$ with $z \cdot p>\operatorname{supp}(p, L)+\varepsilon$ which implies that

$$
\begin{array}{r}
\int_{\Omega} f(x) \mathrm{d} \Phi_{n}^{p}(x)=\operatorname{supp}\left(p, \int_{\Omega} f(x) \mathrm{d} \Phi_{n}(x)\right) \\
<\operatorname{supp}\left(p, \int_{\Omega} f(x) \mathrm{d} \Phi(x)\right)-\varepsilon / 2 \\
=\int_{\Omega} f(x) \mathrm{d} \Phi^{p}(x)-\varepsilon / 2
\end{array}
$$

for any $n$. Now, there is an increasing sequence of simple functions $g_{k}$ which converge upwards to $f$ on $\Omega$. So, by the Monotone Convergence Theorem for finite positive measures we know there is some $N$ so that $k \geq N$ implies that

$$
\int_{\Omega} g_{k}(x) \mathrm{d} \Phi^{p}(x) \geq \int_{\Omega} f(x) \mathrm{d} \Phi^{p}(x)-\varepsilon / 2 .
$$

But then

$$
\begin{array}{r}
\int_{\Omega} g_{k}(x) \mathrm{d} \Phi^{p}(x) \stackrel{n \rightarrow \infty}{\longleftarrow} \int_{\Omega} g_{k}(x) \mathrm{d} \Phi_{n}^{p}(x) \\
<\int_{\Omega} f(x) \mathrm{d} \Phi_{n}^{p}(x)<\int_{\Omega} f(x) \mathrm{d} \Phi^{p}(x)-\varepsilon / 2 \\
\leq \int_{\Omega} g_{k}(x) \mathrm{d} \Phi^{p}(x),
\end{array}
$$

which is a contradiction.

\section{CONE-POSITIVE MULTIMEASURES}

Let $P \subseteq \mathbb{R}^{m}$ be a pointed convex cone. $P$ induces in $\mathbb{R}^{m}$ an order in the usual manner, that is $a \leq_{P} b$ if $b \in a+P$. In a similar way, given $A, B \subseteq R^{m}$ we say $A \leq_{P} B$ if $B \subseteq A+P$; this defines a partial order on subsets of $\mathbb{R}^{m}$. In the following let $P^{*}=\left\{p^{*} \in \mathbb{R}^{m}\right.$ : $\left.p^{*} p \geq 0, \forall p \in P\right\}$ be the dual cone. The following properties are easily proved:

1. $A \subseteq P$ iff $\{0\} \leq{ }_{P} A$

2. $A \subseteq \mathbb{R}^{m}$ and $B \subseteq P$ implies $A \leq A+B$

3. $A, B \subseteq \mathbb{R}^{m}$ and $C \subseteq P$ and $A \leq{ }_{P} B$ implies $A+C \leq_{P}$ $B+C$.

4. $A \subseteq P$ and $q \in P^{*}$ implies that $\operatorname{supp}(q, A) \geq 0$ and $\operatorname{supp}(-q, A) \leq 0$.

5. $A, B \subseteq P$ with $A \leq{ }_{P} B$ implies that $\operatorname{supp}(-q, A) \geq$ $\operatorname{supp}(-q, B)$ for all $q \in P^{*}$.

6. $A+C=B$ with $C \subseteq \mathbb{R}_{+}^{m}$ and $q \in \mathbb{R}_{+}^{m}$ implies that $\operatorname{supp}(q, A) \leq \operatorname{supp}(q, B)$.

We say that a multifunction $F: D \subseteq \mathbb{R}^{n} \rightrightarrows \mathbb{R}^{m}$ is cone-positive if $F(x) \subseteq P$ for all $x \in D$. In a similar way one can define cone-positive multimeasure; given a measurable space $(\Omega, \mathscr{F})$, where $\mathscr{F}$ be a sigmaalgebra defined over $\Omega$, a multimeasure $\Phi: \mathscr{F} \rightrightarrows$ $X$ is said to be cone-positive if $\Phi(A) \subseteq P$ for all $A \in \mathscr{F}$. Let us define $\mathscr{M}_{Q, K}^{++}\left(\Omega, \mathbb{R}^{m}\right)$ be the subspace of cone-positive multimeasures; by using the completeness of $\mathscr{M}_{Q, K}\left(\Omega, \mathbb{R}^{m}\right)$ it is easy to show that $\left(\mathscr{M}_{Q, K}^{++}\left(\Omega, \mathbb{R}^{m}\right), \hat{d}_{M}\right)$ is a complete metric space. We have the following properties:

1. if $A \subseteq B$ then $\Phi(A) \leq{ }_{P} \Phi(B)$, that is $\Phi$ is monotone with respect to $P$

2. if $\Phi$ is a cone-positive multimeasure and $p \in P^{*}$ then $\Phi^{p}$ is a positive measure and $\Phi^{-p}$ is a negative measure

3. if $0 \leq f(x) \leq g(x)$ for all $x$ and $\Phi$ is a cone-positive multimeasure, then $0 \leq \int_{A} f \mathrm{~d} \Phi \leq \int_{A} g \mathrm{~d} \Phi, \forall A$

4. if $F$ is a cone-positive multifunction and $\mu$ is a positive measure then $\Phi(A)=\int_{A} F(x) \mathrm{d} \mu$ defines a cone-positive multimeasure

5. if $\Phi$ is a cone-positive multimeasure and is absolutely continuous with respect to a positive measure $\mu$, then $\mathrm{d} \Phi / \mathrm{d} \mu$ is a cone-positive multifunction

6. if $\Phi$ is cone-positive multimeasure and $f$ is a positive function then $v(A)=\int_{A} f(x) \mathrm{d} \Phi(x)$ is a cone-positive multimeasure. 
Proposition 6. Let $A, B \subseteq P$, convex and compact. Then $A \leq{ }_{P} B$ iff $\forall p \in P^{*}, \operatorname{supp}(-p, A) \geq \operatorname{supp}(-p, B)$.

Proof. Since $A \leq_{P} B$ then we have $B \subseteq A+P$ which implies that $\forall b \in B$ there exists $a \in A$ such that $a \in b$ and then $p(a) \leq p(b)$ for all $p \in P^{*}$. This implies $-p(b) \leq-p(a)$ and then $\operatorname{supp}(-p, A) \geq \operatorname{supp}(-p, B)$. Suppose the converse is not true. Then there exists $\bar{b} \in B$ with $\bar{b} \notin A+P$ and then $a \not \subset \bar{b}$ for all $a \in A$. So for $a \in A, \exists p_{a} \in P^{*}$ such that $p_{a}(a)>p_{a}(b)$, that is $-p_{a}(b)<-p_{a}(a)$. But we assumed $\operatorname{supp}(-p, A) \geq$ $\operatorname{supp}(-p, B)$ so we have a contradiction.

In particular $A \subseteq P$ iff $\operatorname{supp}(-p, A) \leq 0$ for all $p \in P^{*}$.

Proposition 7. Suppose that $\int_{A} F(x) \mathrm{d} \mu(x) \subseteq P$ for all A. Then $F(x) \subseteq P \mu$-a.e.

Proof. For all $p \in P^{*}$ and all $A$ we have that

$$
\int_{A} \operatorname{supp}(-p, F(x)) \mathrm{d} \mu(x) \leq 0,
$$

that is $\operatorname{supp}(-p, F(x)) \leq 0 \mu$-a.e. and then $F(x) \subseteq P$ $\mu$-a.e.

Given two cone-positive multimeasures $\Psi$ and $\Phi$, we say that $\Psi$ is absolutely continuous with respect to $\Phi$ and we write $\Phi \ll \Psi$, iff $\Phi(A)=\{0\}$ implies $\Psi(A)=\{0\}$. It can be easily proved that this is equivalent to require that $\Psi^{p}$ is absolutely continuous with respect to $\Phi^{p}$ for all $p \in P^{*}$.

The following result is an analog of the fact that a positive measure has bounded total mass iff it has bounded variation.

Proposition 8. Let $\Phi$ be a cone-positive multimeasure with convex and compact values in $\mathbb{R}^{d}$. Suppose $P$ is such that $\operatorname{int}\left(P^{*}\right) \neq \emptyset$. Then there exists $K \subseteq P$, compact, such that $\Phi(A) \subseteq K$ for all $A$.

Proof. For all $p \in P^{*}$, we see $\Phi^{p}$ is a positive measure and so $\Phi^{p}(\Omega)=\Phi^{p}(\Omega \backslash A)+\Phi^{p}(A)$ and this implies $\Phi^{p}(A) \leq \Phi^{p}(\Omega)$, that is $\operatorname{supp}(p, \Phi(A)) \leq \operatorname{supp}(p, \Phi(\Omega))$ for all $p \in P^{*}$, $\forall A$. Let $M=\sup _{p \in P^{*},\|p\|=1} \operatorname{supp}(p, \Phi(\Omega))<\infty$ and $K=\bigcap_{p \in P^{*},\|p\|=1}\{x \in P: x \cdot p \leq M\}$. Clearly, $\operatorname{supp}(p, \Phi(\Omega)) \leq M$ for all $p \in P^{*},\|p\|=1$, so $\Phi(A) \subseteq K$, for all $A$. Thus we show $K$ is compact. Suppose not, then there exist $x_{n} \in K$ such that $\left\|x_{n}\right\|>n$. Let $p \in \operatorname{int}\left(P^{*}\right),\|p\|=1$ and $\varepsilon>0$ such that $B_{\varepsilon}(p) \subseteq$ $P^{*}$. Further, let $g_{n} \in \mathbb{R}^{d}$ be such that $\left\|g_{n}\right\|=1$ and $g_{n} x_{n}=\left\|x_{n}\right\|>n$. Then $p+\varepsilon g_{n} \in P^{*}$ and so

$$
M \geq\left(p+\varepsilon g_{n}\right) x_{n}=p x_{n}+\varepsilon g_{n} x_{n}>\varepsilon n-M
$$

which is a contradiction. Thus $K$ is compact.
The following proposition 9 and corollary 2 can be easily proved.

Proposition 9. Suppose that $0 \leq f_{n} \nearrow f$, where $f$ is a bounded function. Suppose further that $\Phi_{n}$ is a sequence of cone-positive multimeasures such that $\Phi_{n}(A) \subseteq \Phi_{n+1}(A), \forall A, \forall n$ with $\Phi_{n}^{p}(\Omega)<M$ for all $n$ and $p \in P^{*}$ with $\|p\|=1$. Let $\Phi(A):=\overline{\bigcup_{n} \Phi_{n}(A)}$. Then $\int f_{n} d \Phi_{n} \rightarrow \int f d \Phi$ in the Hausdorff metric.

Corollary 2. Let $\Phi_{n}$ be a sequence of cone-positive multimeasures and suppose that there is a compact set $K$ with $\Phi_{n}(A) \subseteq \Phi_{n+1}(A), \forall A \subset K, \forall n$. Then $\Phi(A):=\overline{\bigcup_{n} \Phi_{n}(A)}$ is a cone-positive multimeasure and $\int_{A} f d \Phi_{n} \rightarrow \int_{A} f d \Phi$ for all positive function $f$ and $\forall A \subset K$.

\section{SELF-SIMILAR MULTIMEASURES}

Iterated applications of an IFS Markov operator operating on a probability measure converge to a selfsimilar measure which is invariant under the action of the functions in the IFS. We now turn to the definition of self-similarity through an IFS Markov operator on these multimeasures but, to explain this, we give a brief review of the relevant construction. Let $(\Omega, d)$ be a complete metric space and let $\mathscr{F}$ be the corresponding Borel $\sigma$-algebra. Let $w_{i}: \Omega \rightarrow \Omega$ for $i \in\{1,2, \ldots, N\}$ be uniformly contractive. That is, there exists $0<c<1$ such that $d\left(w_{i}(x), w_{i}(y)\right) \leq c d(x, y)$ for each $i$ and all $x, y \in \Omega$. Let $\left(p_{i}\right)_{i=1}^{N}$ be a collection of probabilities such that $p_{i}>0$ and $\sum_{i} p_{i}=1$. This determines the IFS $\left(w_{i}, p_{i}, i=1 \ldots N\right)$. The Markov operator associated with this IFS is an operator on probability measures $\mu$ over $(\Omega, \mathscr{F})$, which is defined by

$$
M \mu(B)=\sum_{i} p_{i} \mu\left(w_{i}^{-1}(B)\right)
$$

for all Borel sets $B \in \mathscr{F}$. If $\mu$ is supported on $B$ and the $w_{i}(B)$ are mutually disjoint then the result of this operator is to assign probability $p_{i}$ to $w_{i}(B)$, that is $M \mu\left(w_{i}(B)\right)=p_{i}$. A second application of $M$ assigns probability $p_{i} p_{j}$ to the set $w_{i}\left(w_{j}(B)\right)$, a third application assigns probability $p_{i} p_{j} p_{k}$ to the set $w_{i}\left(w_{j}\left(w_{k}(B)\right)\right)$, and so on. This recursively partitions a limit probability measure over $\Omega$ which is concentrated on the fractal set defined by the $w_{i}$ 's.

Our intention is to generalize this process to multimeasures; the formulation here of an IFS type method over multimeasures represents recent results from an ongoing research programme concerning the construction of appropriate IFS type 
operators or generalized fractal transforms, including integral and wavelet transforms, over various function spaces, distribution spaces and spaces of generalized measures. Fix two compact and positive sets $K, Q$ (we can consider either notion of positivity) and recall that $\mathscr{M}_{Q, K}\left(\Omega, \mathbb{R}^{m}\right)$ is complete. Let $w_{i}: \Omega \rightarrow \Omega$ for $i=1,2, \ldots, N$ be contractive with the Lipschitz factor for $w_{i}$ being $c_{i}$. We also take linear functions $T_{i}$ : $\mathbb{R}^{m} \rightarrow \mathbb{R}^{m}$ with $\sum_{i} T_{i} Q=Q$ and $\sum_{i \in S} T_{i} K \subseteq K$ for all $S \subseteq\{1,2, \ldots, N\}$ (the choice $K=\lambda Q$ for some $\lambda \geq 1$ often works, but might be overly restrictive). We define the IFS operator

$$
M \Phi(B)=\sum_{i} T_{i}\left(\Phi\left(w_{i}^{-1}(B)\right)\right)
$$

for all $B \in \mathscr{F}$. A simple argument shows that $M \Phi \in$ $\mathscr{M}_{Q, K}\left(\Omega, \mathbb{R}^{m}\right)$ whenever $\Phi \in \mathscr{M}_{Q, K}\left(\Omega, \mathbb{R}^{m}\right)$ (to see this, it is useful to note that each $T_{i}$ is continuous with respect to the Hausdorff metric since $T_{i}$ is linear, and thus Lipschitz).

Theorem 1. For the IFS Markov operator defined above we have

$$
\hat{d}_{M}\left(M \Phi_{1}, M \Phi_{2}\right) \leq\left(\sum_{i} c_{i}\left\|T_{i}\right\|\right) \hat{d}_{M}\left(\Phi_{1}, \Phi_{2}\right)
$$

for all $\Phi_{1}, \Phi_{2} \in \mathscr{M}_{Q, K}\left(\Omega, \mathbb{R}^{m}\right)$.

Proof. First we note that for linear $T$ and convex $A$, we have

$$
\begin{aligned}
& \sup _{q \in S_{1}} \operatorname{supp}(q, T A)=\sup _{q \in S_{1}} \operatorname{supp}\left(T^{*} q, A\right) \\
& \quad \leq\left\|T^{*}\right\| \sup _{q \in S_{1}} \operatorname{supp}(q, A)=\|T\| \sup _{q \in S_{1}} \operatorname{supp}(q, A) .
\end{aligned}
$$

Let $c=\max _{i} c_{i}$ be the contraction factor for the IFS. For a given fixed $q \in S_{1}$ and Lipschitz $f$, we have

$$
\begin{array}{r}
\int_{\Omega} f(x) \mathrm{d}\left[\operatorname{supp}\left(q, M \Phi_{1}(x)\right)-\operatorname{supp}\left(q, M \Phi_{2}(x)\right)\right] \\
=\int_{\Omega} f(x) \mathrm{d}\left[\operatorname{supp}\left(q, \sum_{i} T_{i} \Phi_{1}\left(w_{i}^{-1}(x)\right)\right)\right. \\
\left.-\operatorname{supp}\left(q, \sum_{i} T_{i} \Phi_{2}\left(w_{i}^{-1}(x)\right)\right)\right] \\
=\int_{\Omega} f(x) \sum_{i} \mathrm{~d}\left[\operatorname{supp}\left(T_{i}^{*} q, \Phi_{1}\left(w_{i}^{-1}(x)\right)\right)\right. \\
\left.-\operatorname{supp}\left(T_{i}^{*} q, \Phi_{2}\left(w_{i}^{-1}(x)\right)\right)\right] \\
\leq \int_{\Omega}\left\{\sum_{i}\left\|T_{i}\right\| f\left(w_{i}(y)\right)\right\} . \\
\mathrm{d}\left[\operatorname{supp}\left(q, \Phi_{1}(y)\right)-\operatorname{supp}\left(q, \Phi_{2}(y)\right)\right] .
\end{array}
$$

Now, we see that the function $\tilde{f}=\sum_{i}\left\|T_{i}\right\| f \circ w_{i}$ has Lipschitz factor at most $\sum_{i} c_{i}\left\|T_{i}\right\|$. Thus, when we take the supremum over all $q$ and all Lipschitz functions, we get that

$$
\hat{d}_{M}\left(M \Phi_{1}, M \Phi_{2}\right) \leq\left(\sum_{i} c_{i}\left\|T_{i}\right\|\right) \hat{d}_{M}\left(\Phi_{1}, \Phi_{2}\right),
$$

as was desired.

Example 5.1. As illustrative example, we choose $K=$ $Q \subset \mathbb{R}^{m}$ to be the closed unit ball and $\Omega=[0,1]$ with $w_{i}(x)=x / 2+i / 2$ for $i=0,1$. Further let $p_{0} \in(0,1)$ and $p_{1}=1-p_{0}$ and define $T_{i}=p_{i} I$. Then the invariant multimeasure for the IFS Markov operator

$$
M \phi(B)=T_{0} \phi\left(w_{0}^{-1}(B)\right)+T_{1} \phi\left(w_{1}^{-1}(B)\right)
$$

is the measure $Q \mu$ where $\mu$ is the probability measure which is the invariant distribution for the standard IFS with maps $\left\{w_{0}, w_{1}\right\}$ and probabilities $\left\{p_{0}, p_{1}\right\}$. In this case, the multimeasure is rather simple, being the product of the scalar (probability) measure $\mu$ and the set $Q$. Fig. 1 shows two multimeasure attractors of IFS Markov operators and they have been obtained with the following parameters: $p_{0}=0.3, p_{1}=0.7$ for both of them and, for the rectangular, one direction is 0.3 and 0.7 and the other direction is 0.7 and 0.3 The $w_{i}$ are $x / 2+i / 2, i=0 . .1$.

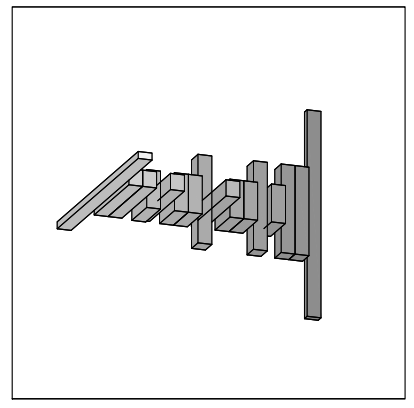

Fig. 1. Rectangular and circular positive multimeasures.

Example 5.2. Let $\Omega=[0,1], w_{0}(x)=x / 3, w_{1}(x)=$ $x / 3+2 / 3\left(\right.$ so $\left.c_{0}=c_{1}=1 / 3\right)$ and

$$
\begin{aligned}
& T_{0}(x, y)=\left(\begin{array}{cc}
\alpha & 0 \\
0 & 1-\alpha
\end{array}\right)\left(\begin{array}{l}
x \\
y
\end{array}\right), \\
& T_{1}(x, y)=\left(\begin{array}{cc}
1-\alpha & 0 \\
0 & \alpha
\end{array}\right)\left(\begin{array}{l}
x \\
y
\end{array}\right),
\end{aligned}
$$

with $1 / 2<\alpha<1$. Now, let $K=Q=[0,1]^{2} \subset \mathbb{R}^{2}$. It is easy to see that $T_{0}(Q)+T_{1}(Q)=Q$. In this case, the invariant multimeasure $\phi$ is supported on the classical Cantor Set and the values are rectangles which are more "vertical" to the left and more "horizontal" to the right. 
Let us suppose that $T_{i}(P) \subseteq P$. This implies that if $B \subseteq P$ then $\sum_{i} T_{i}(B) \subseteq P$. Notice if $T$ satisfies these two hypotheses, then if $\Phi$ is a cone-positive multimeasure with $\Phi(\Omega)=Q$, then $T \Phi(\Omega)=Q$ and $T \Phi$ is a cone-positive multimeasure. Thus $T \Phi(A) \subseteq$ $\bigcap_{p^{*} \in P^{*},\left\|p^{*}\right\|=1}\left\{x: x p^{*} \leq M\right\}$ with the same $M$.

It is also trivial to see that if $\Phi$ is positive in the sense that $0 \in \Phi(A)$ for all $A$, then $M \Phi$ is also positive in this same sense. Thus $M$ preserves both classes of positive multimeasures.

\section{CONCLUSIONS}

At the end of this paper, it is worth spending few words to justify why the concept of positive multimeasure arises quite naturally in the context of fractal image coding. In fact, in the classical IFS coding an image $F$ can be modeled as an $L^{p}$ function or as a positive probability measure. Practically speaking, these are two identical ways to look at a given image; when a function-based representation is used, at each pixel in the domain the function assigns the color corresponding to that pixel. However, when the color of pixel can not be assigned with precision, it might be preferable to use a measure-based approach which assigns the averaged color of a given block of pixels. From a mathematical point of view, there exists a straight correspondence between the above approaches; when a positive function is integrated with respect to the Lebesgue measure, this leads to a positive measure and vice versa, if the the positive measure is absolutely continuous with respect to the Lebesgue measure then, by Radon theorem, there exists a density. In other words these two approaches can be understood as the two faces of the same medal. What happens if we consider a multifunctionbased environment instead? In La Torre et al. (2006) we motivated what are the advantages of using the notion of multifunction in image analysis; roughly speaking this formalism allows to include in a unique framework possible uncertainty or noise on the color of a pixel. In this context, as well described in Fig. 2, the easiest example happens when we consider an interval-valued multifunction, in which the lower and upper functions describe the range of possible color variations.

As it happens for single-valued function, it might happen that the color of a single pixel is difficult to be determined; this leads to consider the measure of a block of pixel and then, quite naturally, to introduce the definition of positive multimeasure.



Fig. 2. Lower and upper Lena images.

\section{REFERENCES}

Aló R, de Korvin A, Roberts Jr. C (1980). On some properties of continuous multimeasures. J Math Anal Appl 75:402-10.

Artstein Z (1972). Set-valued measures. Trans Amer Math Soc 165:103-25.

Barnsley MF (1989). Fractals Everywhere. New York: Academic Press.

Barnsley MF, Demko S (1985). Iterated function systems and the global construction of fractals. Proc Roy Soc London Ser A 399:243-75.

Barnsley MF, Ervin V, Hardin D, Lancaster J (1985). Solution of an inverse problem for fractals and other sets. Proc Natl Acad Sci USA. 83:1975-7.

Brooks JK (1968). An integration theory for set-valued measures. Bull Soc Roy Sci Liege 37(5):312-9.

Choquet G (1953). Theory of capacities. Ann Inst Fourier 5:131-295.

Debreu G, Schmeidler D (1967). The Radon-Nikodym derivative of a correspondence. In: Lecam L, Neyman J, Scott EL, eds. Proc 5th Berkeley Symp Math Stat Probab II (Part 1). Berkeley: University of California Press. 351-72.

Drewnowski L (1976). Additive and countably additive correspondences. Comment Math 19:25-53.

Forte B, Mendivil F, Vrscay ER (1999). IFS operators on integral transforms. In: Dekking M, Levy-Vehel J, Lutton E, Tricot C, eds. Fractals: Theory and Applications in Engineering. London: Springer Verlag.

Forte B, Vrscay ER (1999). Theory of generalized fractal transforms. In: Fisher Y, ed. Fractal Image Encoding and Analysis. NATO ASI Series F. Vol. 159. New York: Springer Verlag.

Guo C, Zhang D (2004). On set-valued fuzzy measures. Inform Sci 160:13-25.

Hildenbrand W (1974). Core and Equilibria of a Large Economy. New York: Princeton University Press. 
Hutchinson J (1981). Fractals and self-similarity. Indiana Univ J Math 30:713-47.

Iacus S, La Torre D (2005). Approximating distribution functions by iterated function systems. J Appl Math Dec Sci 2005(1):33-46..

Iacus S, La Torre D (2005). A comparative simulation study on the IFS distribution function estimator. Nonlinear Anal Real 6:858-73.

Kandilakis D (1992). On the extension of multimeasures and integration with respect to a multimeasure. Proc Am Math Soc 116:85-92.

Kunze H, La Torre D, Mendivil F, Vrscay ER (2012). Fractal-based methods in analysis. New York: Springer.

Kunze H, La Torre D, Vrscay ER (2007). Contractive multifunctions, fixed point inclusions and iterated multifunction systems. J Math Anal Appl 330:157-73.

Kunze H, La Torre D, Vrscay ER (2008). From Iterated Function Systems to Iterated Multifunction Systems. Comm Appl Nonlinear Anal 15:1-15.
La Torre D, Mendivil F, Vrscay ER (2006). Iterated function systems on multifunctions. In: Aletti G et al., eds. Math Everywhere: Deterministic and Stochastic Modeling in Biomedicine, Economics and Industry. New York: Springer Verlag, 125-138.

La Torre D, Mendivil F (2008). Iterated function systems on multifunctions and inverse problems. J Math Anal Appl 340(2):1469-79.

La Torre D, Mendivil F (2009). Union-additive multimeasures and self-similarity. Commun Math Anal 7(2):51-61.

La Torre D, Vrscay ER, Ebrahimi M, Barnsley M (2009). Measure-valued images, associated fractal transforms and the affine self-similarity of images. SIAM J Imaging Sci 2(2):470-507.

Pap E (1991). On non-additive set functions. Atti Sem Mat Fis Univ Modena 39:345-360.

Vind K (1964). Edgeworth-allocations in an exchange economy with many traders. Int Econ Rev 5(2):165177. 